\title{
Fivefold Symmetric Photonic Quasi-Crystal Fiber for Dispersion Compensation from S- to L-Band and Optimized at $1.55 \mu \mathrm{m}$
}

\author{
Sivacoumar Rajalingam and Zachariah C. Alex \\ School of Electronics Engineering, VIT University, Vellore 632014, India \\ Correspondence should be addressed to Sivacoumar Rajalingam; rsivacoumar@vit.ac.in
}

Received 11 May 2015; Accepted 14 July 2015

Academic Editor: Jayanta K. Sahu

Copyright (C) 2015 S. Rajalingam and Z. C. Alex. This is an open access article distributed under the Creative Commons Attribution License, which permits unrestricted use, distribution, and reproduction in any medium, provided the original work is properly cited.

\begin{abstract}
A highly dispersive dual core quasi-periodic photonic crystal fiber is proposed for chromatic dispersion compensation. The dispersion for the dual concentric core fiber is optimized to compensate the chromatic dispersion with a high negative dispersion, accomplishing the communication bandwidth from S-band $(1460 \mathrm{~nm})$ to L-band $(1625 \mathrm{~nm})$. By precise control of structural parameter we have achieved a maximum dispersion of $-18,838 \mathrm{ps} / \mathrm{nm}-\mathrm{km}$ with the phase matching wavelength centred around $1.55 \mu \mathrm{m}$. We also numerically investigate the influence of structural parameter and doping effects and its response on peak dispersion parameter.
\end{abstract}

\section{Introduction}

Photonic crystal fibers (PCFs) are a special class of optical fibers with a significant dielectric layout, which incorporates unique optical properties [1]. Guiding of light is accomplished by a highly regular array of air holes in a background of silica matrix running along the full length of the fiber. PCFs have gained significant interest in researchers due to exceptional optical properties such as wide band single mode operation, flexibility in controlling effective area, nonlinearity, and dispersion parameters. The manipulation of photonic cladding parameters allows the photonic crystal fibers to exhibit unique dispersive properties to be tailored easily for specific applications. These dispersion effects can be used to realize set of functionalities, including beam shaping such as optical pulse stretching [2], supercontinuum generation [3], harmonic generation [4], polarization mode dispersion [5], and dispersion compensation [6-9].

Recent advancement in optical communication networks has progressed towards higher transmission capacity and demand for huge bandwidth has compelled the optical network to adapt Dense Wavelength Division Multiplexing (DWDM) [10]. As the transmission bandwidth is fixed, allowable dispersion values are inversely proportional to the square of the optical pulse compression transmission bit rate speed.
So, the compensation of accumulated chromatic dispersion over transmission bandwidth becomes crucial for optical networks and nonlinear applications. Single-mode fibers, used in high-speed optical networks, are subject to chromatic dispersion that causes pulse broadening depending on wavelength and polarization mode dispersion (PMD) depending on polarization states [5]. The signal degrades and limits the distance a digital signal can travel before needing regeneration or compensation. An optical network operated at $1550 \mathrm{~nm}$ using a standard ITU G.652 fiber having chromatic dispersion of $17 \mathrm{ps} /(\mathrm{nm}-\mathrm{km})$ curtails to a maximum transmission distance of $60 \mathrm{~km}$. Penalties incurred by chromatic dispersion can be minimized using negative dispersion compensating fibers (DCF) [11]. The commercial dispersion compensation fibers for few tens of kilometers are employed for dispersion management usually having negative dispersion in the order of few hundred $\mathrm{ps} /(\mathrm{nm}-\mathrm{km})$. But photonic crystal fibers (PCF) can speculate very high negative dispersion due to high index contrast between core and conformable cladding. PCF structures with high index inner core and a defected ring of reduced holes in the cladding comparable to dual core geometries have accomplished dispersion as high as $-2200 \mathrm{ps} /(\mathrm{nm}-\mathrm{km})[12]$. In this paper, we have proposed a quasi-periodic fiber structure which is self-similar lattice generated by inflation-deflation procedure of matching rules 
with long-range order with no periodicity. It has been evident that quasi-periodic structures can give rise to unusual phenomena and desired properties like large cutoff ratio for endlessly single mode operation, ultraflat dispersion, and so forth, not observed in periodic structures $[8,13,14]$.

This research work targets the design and analysis of a novel type of dual core PQF. First, it is demonstrated by befitting structural and material parameters, and then the geometrically modified lattice structure may result in very high values of negative dispersion parameter within the spectral range of optical fiber communications windows. Next, a parametric investigation is carried out focusing on the impact of various structural [15] and material parameters [16] optimizing on the dispersion profile of the proposed PQF.

\section{Theory and Design of a Dual-Concentric-Core PCF}

We have illustrated the transverse cross section of 5-fold DCPQF in Figure 1. Quasi-crystals are crystals in which they have long-range translational and orientational orders. However, the translational order is not periodic and the structure does not necessarily have crystallographic rotational point symmetry. These structures reveal more significant attributes than the stereotyped PCFs, due to the degree of freedom that has been concealed in aperiodic structures. The proposed $\mathrm{PQF}$ structure is formed by two types of rhombic tiles, thin tile with angles of $36^{\circ}$ and $144^{\circ}$, and thick tile with angles of $72^{\circ}$ and $108^{\circ}$. The resulting connected space-filling packing of unit cells is called Penrose lattice. The inner core and the surrounding air holes are constructed on the base of 2dimensional Penrose lattice. In our analysis the refractive index of fused silica as a function $\lambda$ is approximated using three-term Sellmeier equation [17]:

$$
n^{2}(\lambda)=1+\sum_{i=1}^{3} \frac{a_{i} \lambda^{2}}{\lambda^{2}-b_{i}^{2}},
$$

where $\lambda$ is the wavelength, $n$ is the refractive index as a function of $\lambda, a_{i}$ is the oscillator strength, and $b_{i}$ is the oscillator resonance frequency. In an index-guiding PCF, the core refractive index is greater than the average index of the cladding, since the air holes are interweaved in the silica matrix, and the fiber can guide the light by total internal reflection as a standard single mode fiber does. That is, the guided light has an effective index $n_{\text {eff }}$ that satisfies the condition $n_{\text {core }}>n_{\text {eff }}=\left(\beta / k_{0}\right)$ [18]. As per the theoretical aspects discussed the proposed $\mathrm{PQF}$ is designed such that inner core of PQF is with a diameter of $1.7 \mu \mathrm{m}$ and $\left(d_{\text {core }}\right)$ is doped with very low concentration of Germanium contributing an index difference of $2.75 \%$. The outer defected core is a pure fused silica core formed by eliminating the second ring of 15 air holes. Inner cladding is formed between the two cores with hole diameter of $2.2 \mu \mathrm{m}\left(d_{1}\right)$. We have maintained $2.3 \mu \mathrm{m}$ between cores to first cladding ring and thereby the lattice constant or pitch $(\Lambda)$ was maintained to be $2.3 \mu \mathrm{m}$. In this design, we have designated outer cladding air holes with two different diameters $1.8 \mu \mathrm{m}\left(d_{2}\right)$ and $0.7 \mu \mathrm{m}\left(d_{3}\right)$. The air hole in the cladding depresses the average index of

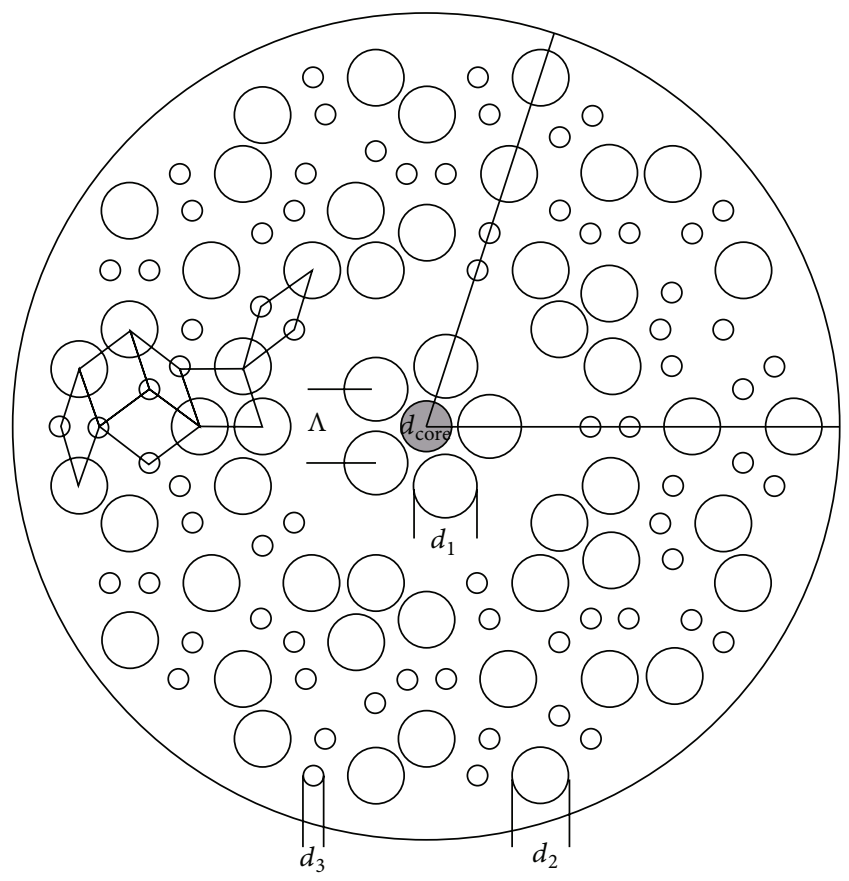

FIGURE 1: Cross section of the proposed 5-fold DC-PQF.

the cladding region and confines light within the central core. The highest negative dispersion can be tailored with suitable design of these three parameters $d_{1}, d_{2}$, and $d_{3}$ and with careful doping concentration of core diameter the phase matching peak wavelength can be shifted to a commodious value. The air filling ratio of the lattice with $0.96\left(d_{1} / \Lambda\right)$, $0.87\left(d_{2} / \Lambda\right)$, and $0.27\left(d_{3} / \Lambda\right)$ for inner-core and the outer-core is maximized by the quasi-lattice distribution.

The calculation of the dispersion properties of PCF modes requires a highly efficient numerical method. At present, the modal solution approach based on the FEM is more flexible than most approaches as it matches well with the complex structural properties of PCFs such as different photonic-crystal lattice geometries, nonperfect lattices (PQF) and holes, asymmetries, and different materials. In this research work we employed finite element method (FEM) with anisotropic perfectly matched layers (PML) to assess the effective index of the core mode in PQF.

The PQF cross section shown in Figure 1 with a finite number of air holes is divided into homogeneous subspaces where Maxwell's equations are solved by accounting for the adjacent subspaces. These subspaces are triangles that allow a good approximation of the circular structures. Using the anisotropic PML from the Maxwell equations the following vectorial equation is derived [19]:

$$
\nabla \times\left([\mu]_{\mathrm{PML}}^{-1} \nabla \times \vec{E}\right)-k_{0}^{2}[\varepsilon]_{\mathrm{PML}} \vec{E}=0
$$

where $k_{0}$ is the free space wavenumber and $\mathbf{E}$ is the electric field vector. The PML can have arbitrary thickness and is specified to be absorbing materials. The materials have anisotropic permittivity $[\mu]_{\mathrm{PML}}$ and permeability $[\varepsilon]_{\mathrm{PML}}$ that 
match the physical medium outside the PML in such a way there are no reflections at the boundaries:

$$
\begin{aligned}
\mu_{[\mathrm{PML}]} & =\mu_{r} \mu_{0} L, \\
\varepsilon_{[\mathrm{PML}]} & =\varepsilon_{0} \varepsilon_{r} L, \\
L & =\left[\begin{array}{ccc}
L_{x x} & 0 & 0 \\
0 & L_{y y} & 0 \\
0 & 0 & L_{z z}
\end{array}\right],
\end{aligned}
$$

where $\mathbf{L}$ is a rank 2 tensor. For a PML which is parallel to one of the Cartesian coordinate planes, $\mathbf{L}$ becomes diagonal, which is given by

$$
\begin{aligned}
& L_{x x}=\frac{s_{y} s_{z}}{s_{x}}, \\
& L_{y y}=\frac{s_{z} s_{x}}{s_{y}}, \\
& L_{z z}=\frac{s_{x} s_{y}}{s_{z}} .
\end{aligned}
$$

The parameters $s_{x}, s_{y}$, and $s_{z}$ are the complex valued coordinate scaling parameters. By assigning suitable values, the PML can absorb the waves travelling in a particular direction. The real part of $s_{x}, s_{y}$, and $s_{z}$ affects how fast an evanescent wave decays in the PML region [19].

The concept of a dual concentric-core PCF is similar to that of a directional coupler which can be analyzed using the coupled mode theory. The guided modes in PCF get localized due to the defected lattices and their model fields are well localized by Hermitian-Gaussian function [18]. The central core and the outer core are defected lattices which behave as two parallel waveguides, and the high dispersion results from the coupling between the two waveguides. It is found that the effective refractive index of the inner-core mode and outer-core mode matches with each other at phase matching wavelength $\left(\lambda_{p}\right)$ (Figure $2(\mathrm{~b})$ ). Before the phase matching wavelength (Figure $2(\mathrm{a}))\left(\lambda<\lambda_{p}\right)$, the field distribution of the inner core mode is confined within the central core and is a Gaussian shape. After the phase matching wavelength $(\lambda>$ $\lambda_{p}$ ) (Figure 2(c)), the fundamental mode field distribution is in the outer core region. Due to the redistribution of modal fields, there is a rapid change in the slope of effective index around the phase matching wavelength, leading to a large negative dispersion $[9,20,21]$.

The dispersion of the PQF can be expressed as the sum of material dispersion and waveguide dispersion approximately. As the effective refractive index $\left(n_{\text {eff }}\right)$ is estimated as a function of wavelength, then dispersion parameter can be computed from the following equation:

$$
D(\lambda)=-\left(\frac{\lambda}{c}\right)\left(\frac{d^{2} n_{\mathrm{eff}}}{d \lambda^{2}}\right)=-\left(\frac{2 \pi c}{\lambda^{2}}\right)\left(\frac{d^{2} \beta}{d \omega^{2}}\right) .
$$

The dispersion value becomes maximum if the wavelength matches the phase matching wavelength [22]:

$$
\begin{aligned}
D_{\max } & = \pm\left(\frac{\pi}{2 c \kappa}\right)\left(\frac{d n_{1}}{d \lambda}-\frac{d n_{2}}{d \lambda}\right)^{2}, \\
\Delta \lambda & =0.766 \times\left(\frac{2 \kappa \lambda_{p}}{\pi}\right)\left|\left(\frac{d n_{1}}{d \lambda}-\frac{d n_{2}}{d \lambda}\right)^{-2}\right| .
\end{aligned}
$$

We can infer from the above equations that the peak dispersion totally depends on the coupling coefficient $\kappa$ and the index slope between the inner-core mode and the cladding mode at phase matching wavelength $\left(\lambda_{p}\right)$. From (6) it is evident that if the inner-core mode and the outer-core mode are easy to couple, a big coupling constant $\kappa$ is obtained, leading to a low peak dispersion value and a broad FWHM. Instead, if there is a hard coupling between the two modes, $\kappa$ is small, leading to a high peak dispersion value and a narrow FWHM [22]. It is observed from Figure 2(d) fundamental mode field distribution confined within the core and the second order mode trapped within the outer core twitch towards each other as the operating wavelength changes. It is indisputable that at a point of inflexion (Figure $2(\mathrm{~d}))\left(\lambda_{p}=\right.$ $1.55 \mu \mathrm{m})$ slope of effective refractive index changes due to coupling of mode energy from inner to outer and contrariwise. At phase matching condition the effective mode field area of $3.4 \mu \mathrm{m}^{2}$ inflates to a very large value of $184.76 \mu \mathrm{m}^{2}$. The splicing loss with standard SMF28 is minimized to a large extent as the effective mode area is in agreement with single mode fiber. After the phase matching wavelength $1.55 \mu \mathrm{m}$ the inner core fundamental mode totally relocates to outer core and vice versa. Even though the analysis is carried out for many phase matching wavelengths, for discussion purpose $1.55 \mu \mathrm{m}$ is taken into account. The modes of single-material PCFs are inherently leaky because the core index is the same as the index of the outer cladding region without air holes. Since in our proposed design the core is doped with an index difference of $2.75 \%$ of $\mathrm{Ge}$, the confinement loss is reduced as the index difference enhances the guiding modes. The confinement loss is deduced from the value of $n_{\text {eff }}$ as

$$
\text { Confinement Loss }=8.686 \operatorname{Im}\left[k_{0} n_{\text {eff }}\right]
$$

in $\mathrm{dB} / \mathrm{m}$, where Im stands for the imaginary part of effective refractive index. The confinement loss was $3.6 \times 10^{-6} \mathrm{~dB}$ for inner-core mode and increases to $1.02 \times 10^{-3} \mathrm{~dB}$ for the combined mode but still the loss is computed to be on the lower side.

\section{Analysis of Geometric Parameter Influences on Optical Property}

It is noteworthy to disclose the fact that material dispersion strongly affects the performance of PCF-based devices designed to operate in a low-dispersion regime, with a significant effect on total device dispersion. The material dispersion also changes with the type of dopant used during glass fabrication. Therefore, it becomes quite crucial to investigate 


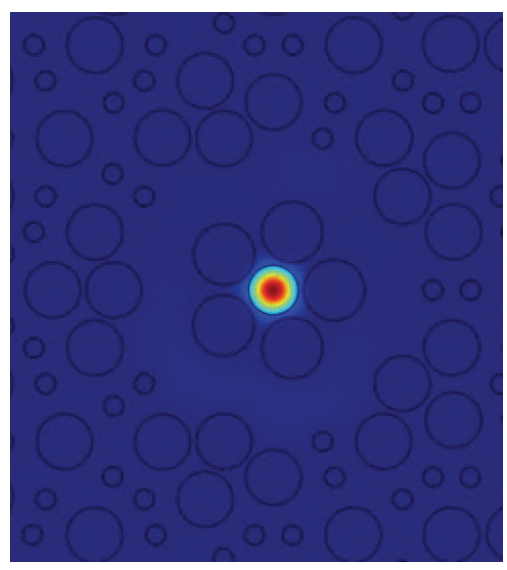

(a)

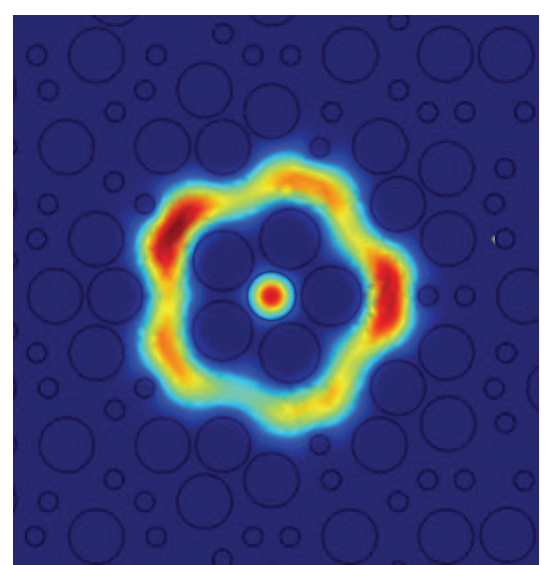

(b)

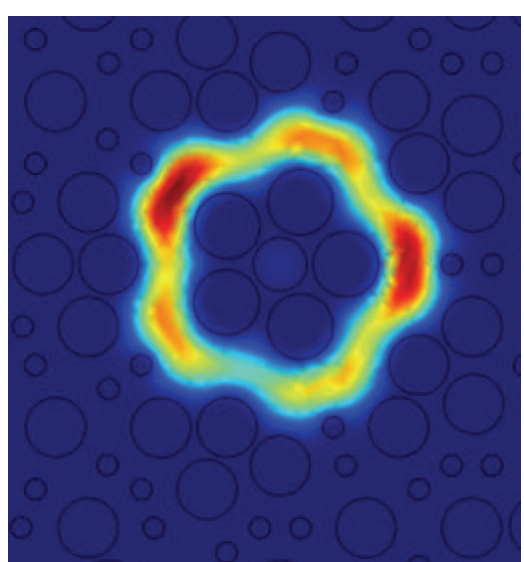

(c)

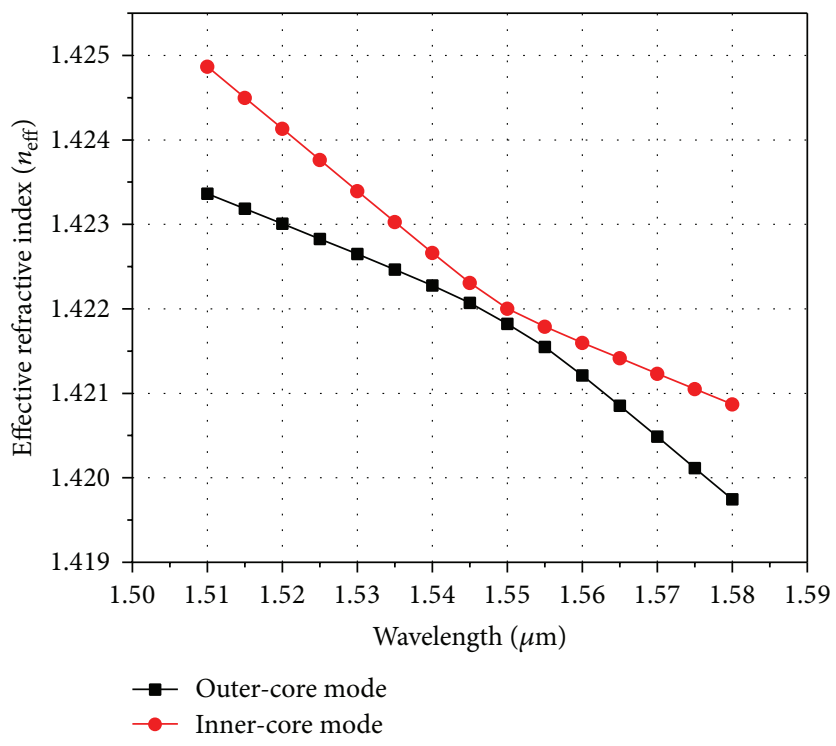

(d)

FIGURE 2: Mode confinement in PQF (a) inner-core mode, (b) combined inner and outer cladding mode, (c) outer cladding mode, and (d) variation of effective index with wavelength for DC-PQF, a curve with circle corresponds to fundamental core mode and square symbol curve represents outer cladding mode.

how different percentage of doped silica glasses affects the PCF response. In our analysis, we have investigated that the phase-matching wavelength shifts as a function of doping percentage, further the radius of first cladding ring is varied showing a very high deviation in the dispersion parameter. From Figure 3(a) it is evident that when $d_{1}=2.2 \mu \mathrm{m}, d_{2}=$ $2.0 \mu \mathrm{m}, d_{3}=0.7 \mu \mathrm{m}$, and $d_{\text {core }}=0.85 \mu \mathrm{m}$, the phase matching wavelength is at $1.54 \mu \mathrm{m}$ for an index difference of $2.71 \%$ with dispersion parameter reaching $-17,312 \mathrm{ps} /(\mathrm{nm}-\mathrm{km})$. When index difference is increased, the phase matching value relocates towards higher wavelength of $1.55 \mu \mathrm{m}$ for $2.75 \%$. The dispersion value for the above index variation falls at $-17,312 \mathrm{ps} /(\mathrm{nm}-\mathrm{km})$ to $-18,838 \mathrm{ps} /(\mathrm{nm}-\mathrm{km})$, respectively, for index variation of $2.71 \%$ to $2.75 \%$.

We have considered influence of core diameter on the shift in the dispersion property [23]. Keeping the doping percentage constant at $2.75 \%$ and $d_{1}=2.2 \mu \mathrm{m}, d_{2}=2.0 \mu \mathrm{m}$, and $d_{3}=0.7 \mu \mathrm{m}$ the core diameter was varied from $0.8 \mu \mathrm{m}$ to $0.9 \mu \mathrm{m}$ in steps of $0.025 \mu \mathrm{m}$. From Figure 3(b), we can apprehend that the phase matching wavelength shifts to a greater extent with maximum dispersion of $-21,619 \mathrm{ps} /(\mathrm{nm}$ $\mathrm{km})$ at $1.50 \mu \mathrm{m}$ to $-17,168 \mathrm{ps} /(\mathrm{nm}-\mathrm{km})$ at $1.595 \mu \mathrm{m}$. This result implies that PCF fabrication must be carefully controlled in order to keep the core radius as close as possible to the designed value at desired wavelength [24].

Eventually the structural variation of $d_{1}, d_{2}$, and $d_{3}$ also has influence on the peak dispersion and phase matching wavelength. Keeping the doping percentage constant at $2.75 \%$ and $d_{\text {core }}=0.85 \mu \mathrm{m}, d_{2}=2.0 \mu \mathrm{m}$, and $d_{3}=0.7 \mu \mathrm{m}$ the $d_{1}$ diameter was varied from $2.1 \mu \mathrm{m}$ to $2.3 \mu \mathrm{m}$ in steps of $0.05 \mu \mathrm{m}$ (Figure 4(a)), and the dispersion parameter takes high values of $-24,356 \mathrm{ps} /(\mathrm{nm}-\mathrm{km})$ and decreases to $-9,121 \mathrm{ps} /(\mathrm{nm}-\mathrm{km})$, 


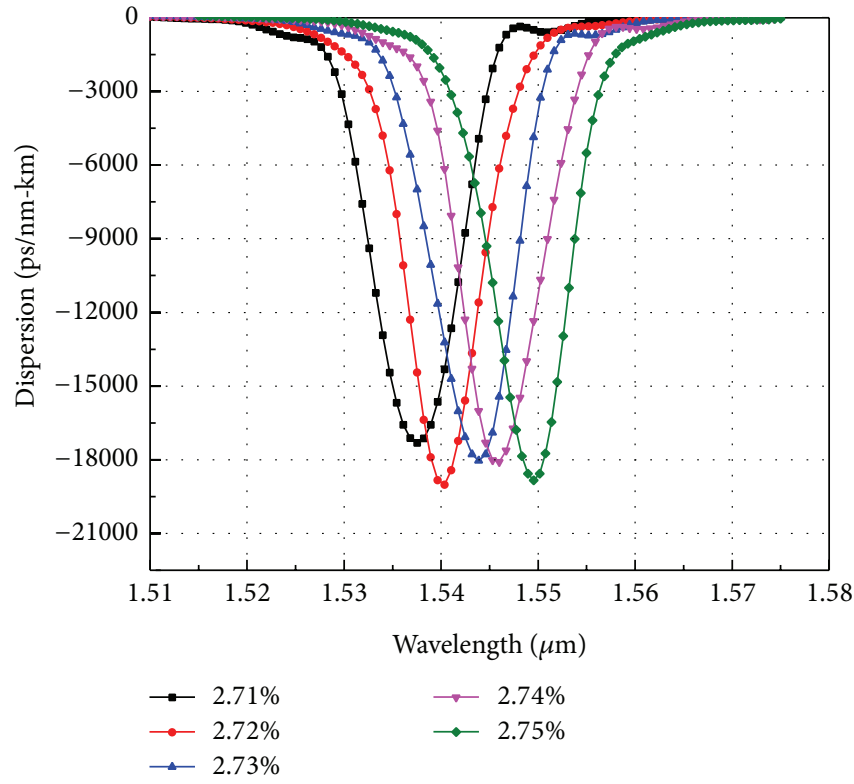

(a)

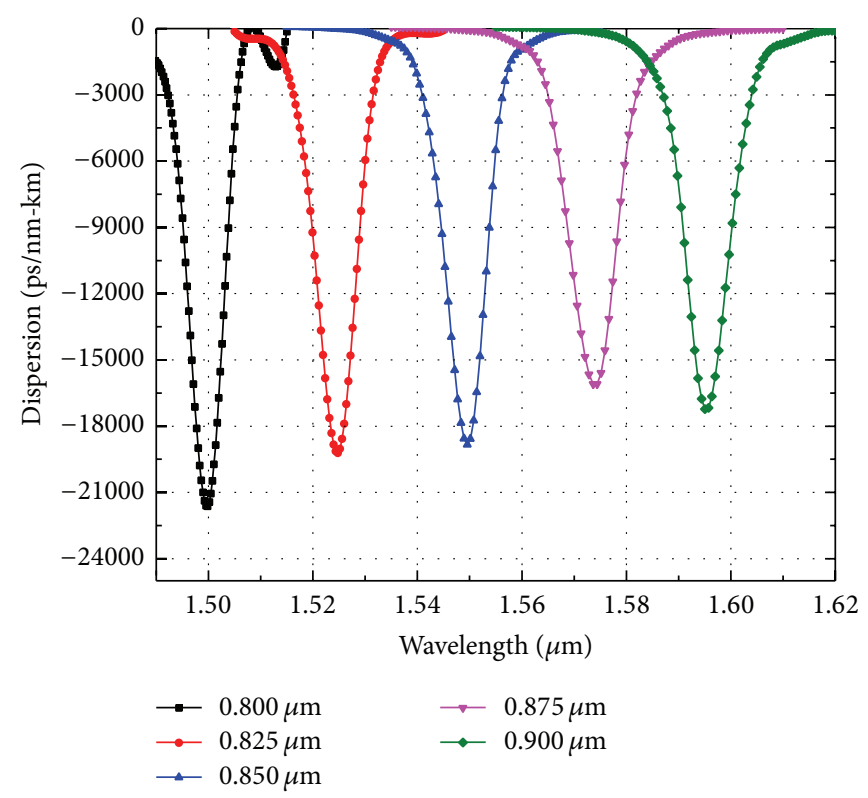

(b)

FIgURE 3: Variation in dispersion parameter due to (a) Core Doping Percentage and (b) core radius.

but the phase matching wavelength increases from to $1.49 \mu \mathrm{m}$ to $1.61 \mu \mathrm{m}$. Interestingly the phase matching wavelength gets perturbed even with variation of $d_{2}$ diameter. Preserving $d_{\text {core }}=0.85 \mu \mathrm{m}, d_{1}=2.2 \mu \mathrm{m}$, and $d_{3}=0.7 \mu \mathrm{m}$ the $d_{2}$ diameter was varied from $1.9 \mu \mathrm{m}$ to $2.1 \mu \mathrm{m}$ in steps of $0.05 \mu \mathrm{m}$. It was recognized from Figure 4(b) that the shift in the phase matching wavelength was not pronounced as much as compared to the diameter variation of $d_{1}$ parameter. The dispersion was downsizing as the $d_{2}$ diameter increases, but a minimum dispersion of $-15,818 \mathrm{ps} / \mathrm{nm}-\mathrm{km}$ occurred for $d_{2}=$ $2.10 \mu \mathrm{m}$.

Similarly, maintaining other parameters constant $d_{3}$ was altered from $0.7 \mu \mathrm{m}$ to $1.1 \mu \mathrm{m}$. From the analysis shown in the Figure 4(c) maximum dispersion of $-18,838 \mathrm{ps} /(\mathrm{nm}-\mathrm{km})$ is occurring at $0.7 \mu \mathrm{m}$ and decreases as the $d_{3}$ progresses. In the present proposed PQF design, the numbers of holes with diameters $d_{3}, d_{2}$, and $d_{1}$ were 70,50 , and 5 , respectively. The dispersion properties and phase matching wavelength of the PQF vary as a function of air filling fraction, contributed by different composition of air holes diameter. The optimized dispersion was found to be maximum at $1.55 \mu \mathrm{m}$ with $-18,838 \mathrm{ps} /(\mathrm{nm}-\mathrm{km})$ for $d_{1}=2.2 \mu \mathrm{m}, d_{2}=1.9 \mu \mathrm{m}$, and $d_{3}=$ $0.8 \mu \mathrm{m}$. From the above analysis, we have to underline the fact that the bandwidth is narrow when dispersion parameter was at its highest value and increased as the dispersion decreased. There is generally a trade-off between the highest dispersion with bandwidth, which can be optimized by careful selection of holes diameter [22]. Geometrical imperfection of a PQF can depreciate its performance. The PQF can be fabricated by the complex fabrication method such as sol-gel methods [25, 26], not by prevailing stacking method, because the quasiperiodic orientation cannot be perfected by the stacking method. The sol-gel method naturally gives well-arranged air holes. As a casting method, the sol-gel technique can fabricate any structure, which can be integrated into a mold. The hole size, shape, and spacing may all be adjusted individually. By comparison, stack and draw methods are constrained to closest-packed design such as triangular or honeycomb lattices. Drilling methods allow adjustment of both the hole size and spacing but are generally bounded to a small number of holes and limited to circular shapes. On the contrary, drilling of preforms leaves roughened surfaces along the side walls of the air hole. Extrusion techniques confer design freedom but are often limited to soft glasses for which the material loss values are exceedingly high $[27,28]$.

\section{Conclusion}

In this paper, we have thoroughly explored the possibilities of various structural parameters and their influences on the peak dispersion parameter conjointly with shift in phase matching wavelength. By varying the sizes of air holes, we obtained a maximum negative dispersion of $-24,356 \mathrm{ps} /(\mathrm{nm}-$ $\mathrm{km})$ for our proposed PQF. The design was optimized for $-18,838 \mathrm{ps} /(\mathrm{nm}-\mathrm{km})$ at phase matching wavelength of $1.55 \mu \mathrm{m}$. We conclude, with optimized structural parameters and with low confinement loss, that our proposed fivefold symmetric dual core PQF is envisaged as a dispersion compensating fiber.

\section{Conflict of Interests}

The authors declare that there is no conflict of interests regarding the publication of this paper. 


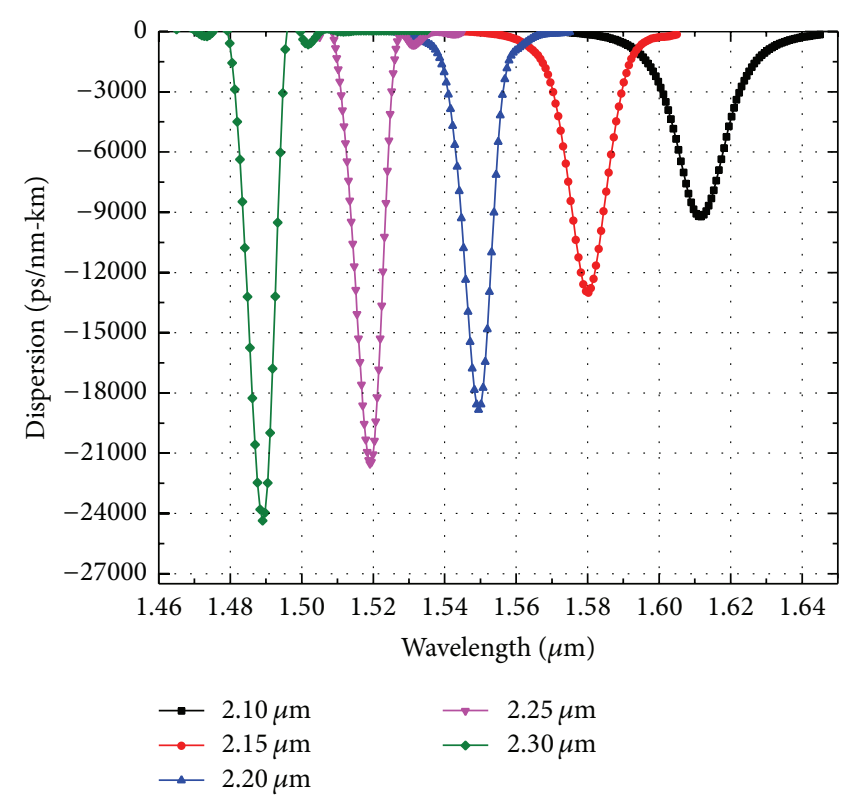

(a)

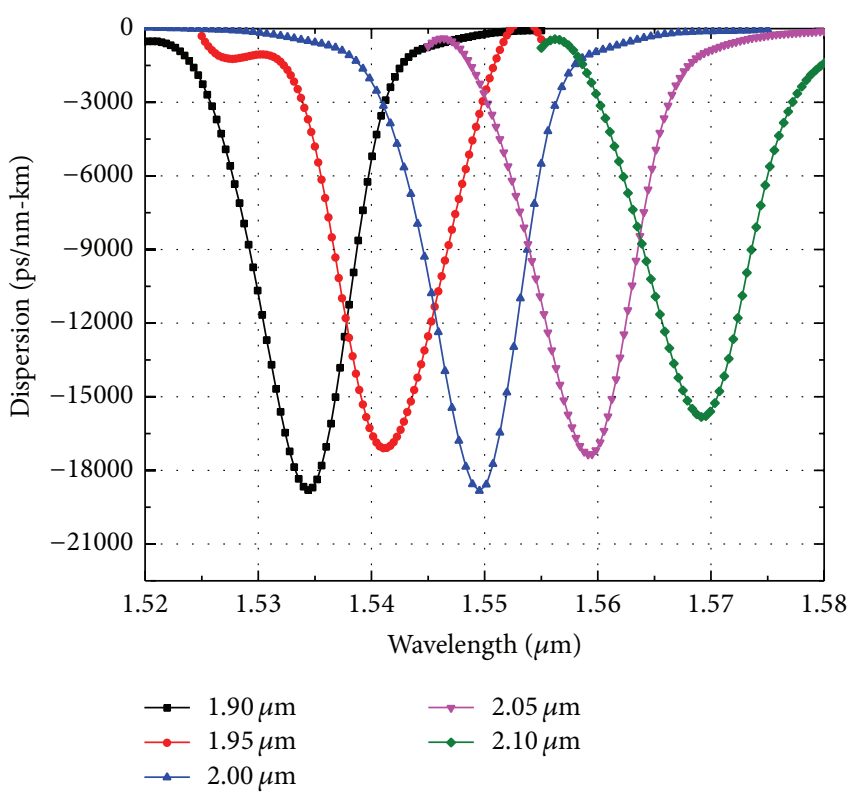

(b)

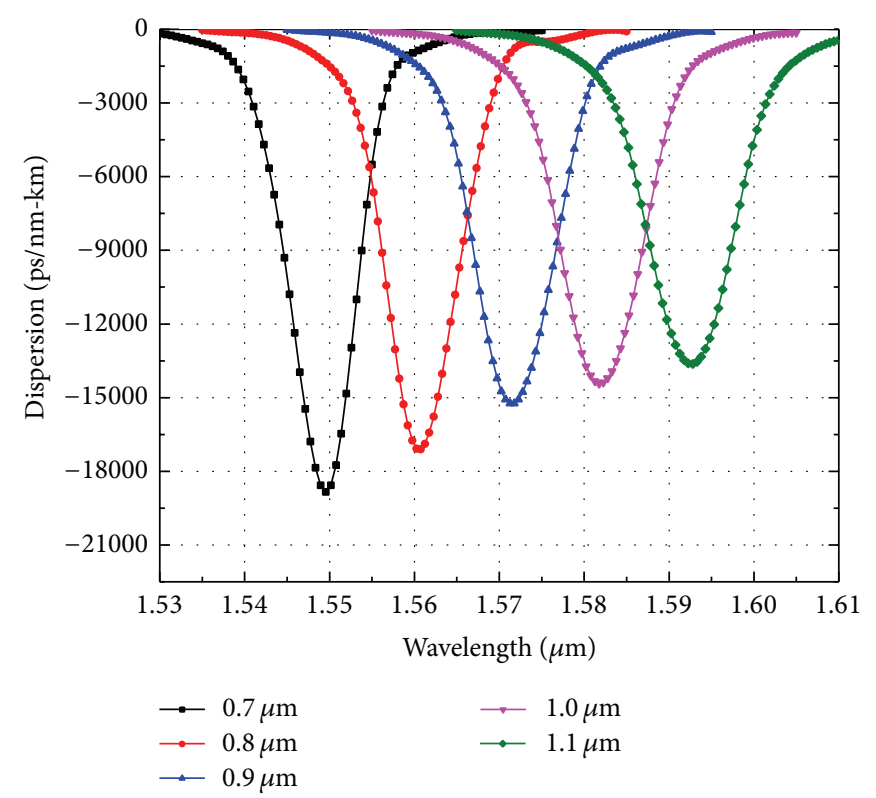

(c)

FIgURE 4: Variation in dispersion parameter due to (a) only $d_{1}$ hole variation, (b) only $d_{2}$ hole variation, and (c) only $d_{3}$ hole variation.

\section{References}

[1] T. A. Birks, J. C. Knight, and P. S. J. Russell, "Endlessly singlemode photonic crystal fiber," Optics Letters, vol. 22, no. 13, pp. 961-963, 1997.

[2] D. G. Ouzounov, C. J. Hensley, A. L. Gaeta, N. Venkateraman, M. T. Gallagher, and K. W. Koch, "Soliton pulse compression in photonic band-gap fibers," Optics Express, vol. 13, no. 16, pp. 6153-6159, 2005.

[3] T. A. Birks, W. J. Wadsworth, and P. S. J. Russell, "Supercontinuum generation in tapered fibers," Optics Letters, vol. 25, no. 19, pp. 1415-1417, 2000.
[4] T. Schreiber, J. Limpert, H. Zellmer, A. Tünnermann, and K. P. Hansen, "High average power supercontinuum generation in photonic crystal fibers," Optics Communications, vol. 228, no. 13, pp. 71-78, 2003.

[5] D. C. Zografopoulos, C. Vázquez, E. E. Kriezis, and T. V. Yioultsis, "Dual-core photonic crystal fibers for tunable polarization mode dispersion compensation," Optics Express, vol. 19, no. 22, pp. 21680-21691, 2011.

[6] K. Saitoh, M. Koshiba, T. Hasegawa, and E. Sasaoka, "Chromatic dispersion control in photonic crystal fibers: application to ultra-flattened dispersion," Optics Express, vol. 11, no. 8, pp. 843852, 2003. 
[7] X. Zhao, G. Zhou, L. Shuguang et al., "Photonic crystal fiber for dispersion compensation," Applied Optics, vol. 47, no. 28, pp. 5190-5196, 2008.

[8] S. Kim and C.-S. Kee, "Dispersion properties of dual-core photonicquasicrystal fiber," Optics Express, vol. 17, no. 18, pp. 15885-15890, 2009.

[9] Y.-H. Li, W.-D. Fan, and Q.-Q. Sheng, "A novel photonic quasicrystal fiber with broadband large negative dispersion," Chinese Physics Letters, vol. 27, no. 11, Article ID 114211, 2010.

[10] F. Begum, Y. Namihira, S. F. Kaijage et al., "Design of broadband dispersion compensating photonic crystal fibers for high speed transmission systems," in Proceedings of the Optical Fiber Communication Conference and National Fiber Optic Engineers Conference, OSA Technical Digest, 2009.

[11] K. Thyagarajan, R. K. Varshney, P. Palai, A. K. Ghatak, and I. C. Goyal, "A novel design of a dispersion compensating fiber," IEEE Photonics Technology Letters, vol. 8, no. 11, pp. 1510-1512, 1996.

[12] F. Gérôme, J.-L. Auguste, and J.-M. Blondy, "Design of dispersion-compensating fibers based on a dual-concentric-core photonic crystal fiber," Optics Letters, vol. 29, no. 23, pp. 27252727, 2004.

[13] X. Sun and D. J. J. Hu, "Air guiding with photonic quasi-crystal fiber," IEEE Photonics Technology Letters, vol. 22, no. 9, pp. 607609, 2010.

[14] M. Mejbaul Haque, M. Shaifur Rahman, M. Selim Habib, and M. Samiul Habib, "A single mode hybrid cladding circular photonic crystal fiber dispersion compensation and sensing applications," Photonics and Nanostructures-Fundamentals and Applications, vol. 14, pp. 63-70, 2015.

[15] S. F. Kaijage, Y. Namihira, N. H. Hai et al., "Broadband dispersion compensating octagonal photonic crystal fiber for optical communication applications," Japanese Journal of Applied Physics, vol. 48, no. 5, 2009.

[16] M. Samiul Habib, K. M. Nasim, M. Selim Habib, M. Imran Hasan, and R. Ahmad, "Relative dispersion slope matched dispersion compensating highly birefringent spiral microstructure optical fibers using defected core," Optical Engineering, vol. 52, no. 9, Article ID 130943, 2013.

[17] B. Tatian, "Fitting refractive-index data with the Sellmeier dispersion formula," Applied Optics, vol. 23, no. 24, pp. 44774485, 1984.

[18] K. Saitoh and M. Koshiba, "Numerical modeling of photonic crystal fibers," Journal of Lightwave Technology, vol. 23, no. 11, pp. 3580-3590, 2005.

[19] C.-P. Yu and H.-C. Chang, "Yee-mesh-based finite difference eigenmode solver with PML absorbing boundary conditions for optical waveguides and photonic crystal fibers," Optics Express, vol. 12, no. 25, pp. 6165-6177, 2004.

[20] A. Huttunen and P. Törmä, "Optimization of dual-core and microstructure fiber geometries for dispersion compensation and large mode area," Optics Express, vol. 13, no. 2, pp. 627-635, 2005.

[21] S. Rajalingam and Z. C. Alex, "Five-fold symmetric photonic quasi-crystal fiber with high negative dispersion," Research Journal of Applied Sciences, Engineering and Technology, vol. 9, no. 9, pp. 786-791, 2015.

[22] H. Subbaraman, T. Ling, Y. Jiang, M. Y. Chen, P. Cao, and R. T. Chen, "Design of a broadband highly dispersive pure silica photonic crystal fiber," Applied Optics, vol. 46, no. 16, pp. 32633268, 2007.
[23] L. P. Shen, W.-P. Huang, G. X. Chen, and S. S. Jian, "Design and optimization of photonic crystal fibers for broad-band dispersion compensation," IEEE Photonics Technology Letters, vol. 15, no. 4, pp. 540-542, 2003.

[24] C.-P. Yu, J.-H. Liou, S.-S. Huang, and H.-C. Chang, "Tunable dual-core liquid-filled photonic crystal fibers for dispersion compensation," Optics Express, vol. 16, no. 7, pp. 4443-4451, 2008.

[25] Y. D. Hazan, J. B. MacChesney, T. E. Stockert, D. J. Trevor, and R. S. Windeler, "Sol-gel method of making an optical fiber with multiple apertures," US Patent 6467312 B1, 2000.

[26] R. T. Bise and D. J. Trevor, "Sol-gel derived microstructured fiber: fabrication and characterization," in Proceedings of the Optical Fiber Communication Conference, Technical Digest (OFC/NFOEC '05), March 2005.

[27] H. Ebendorff-Heidepriem and T. M. Monro, "Extrusion of complex preforms for microstructured optical fibers," Optics Express, vol. 15, no. 23, pp. 15086-15092, 2007.

[28] T. Yajima, J. Yamamoto, F. Ishii, T. Hirooka, M. Yoshida, and M. Nakazawa, "Low-loss photonic crystal fiber fabricated by a slurry casting method," Optics Express, vol. 21, no. 25, pp. 3050030506, 2013. 

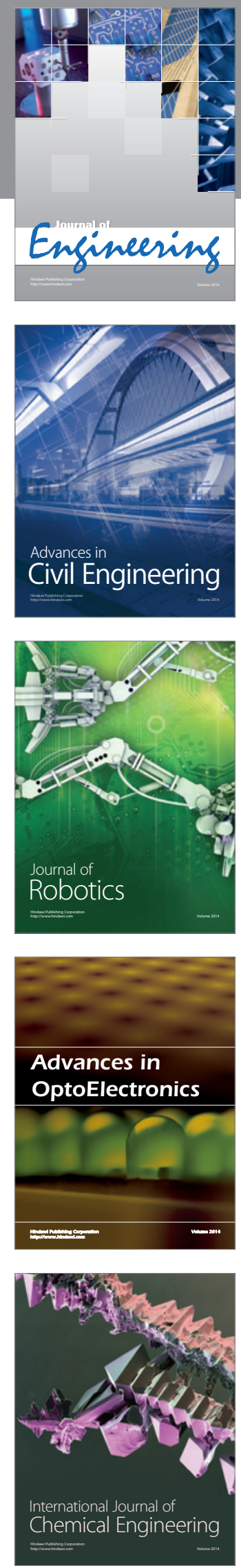

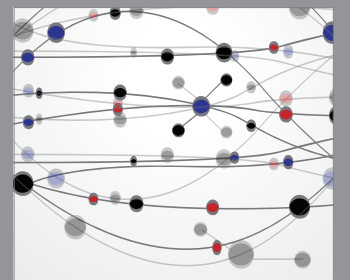

The Scientific World Journal
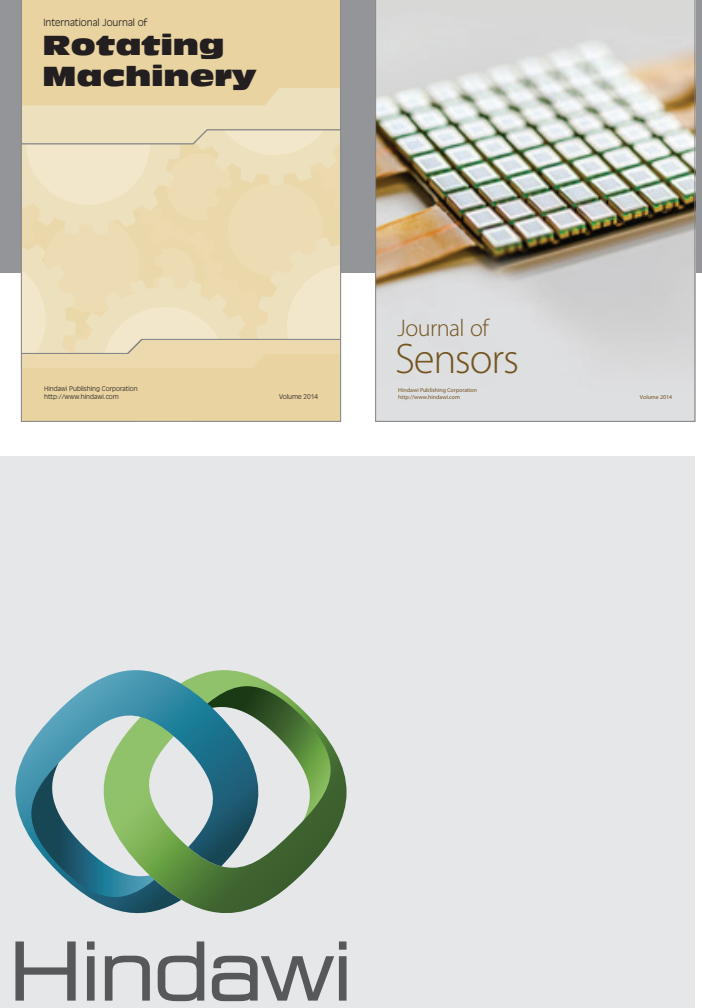

Submit your manuscripts at http://www.hindawi.com
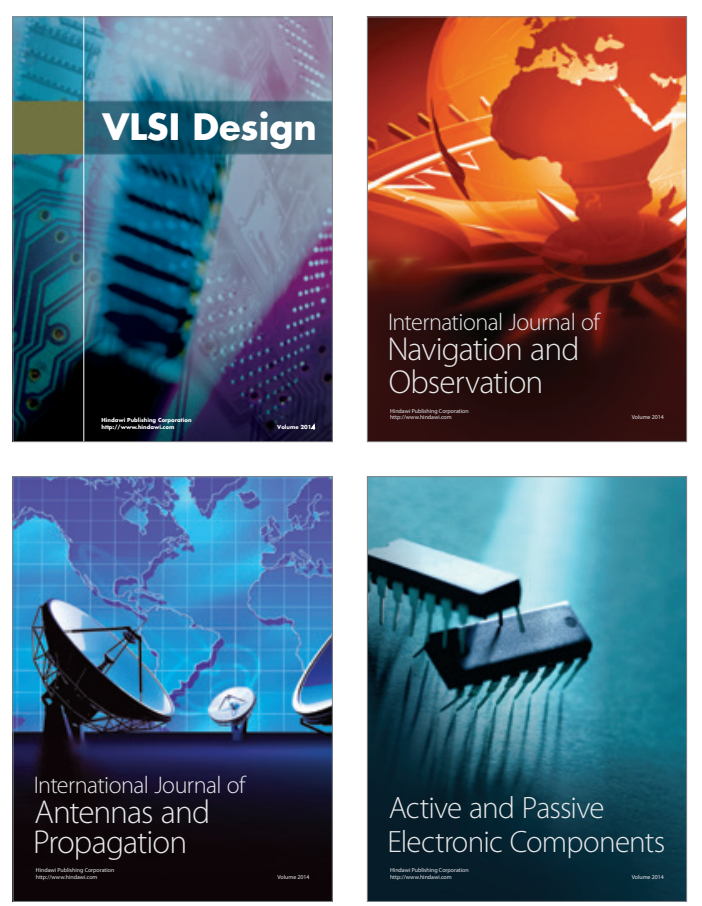
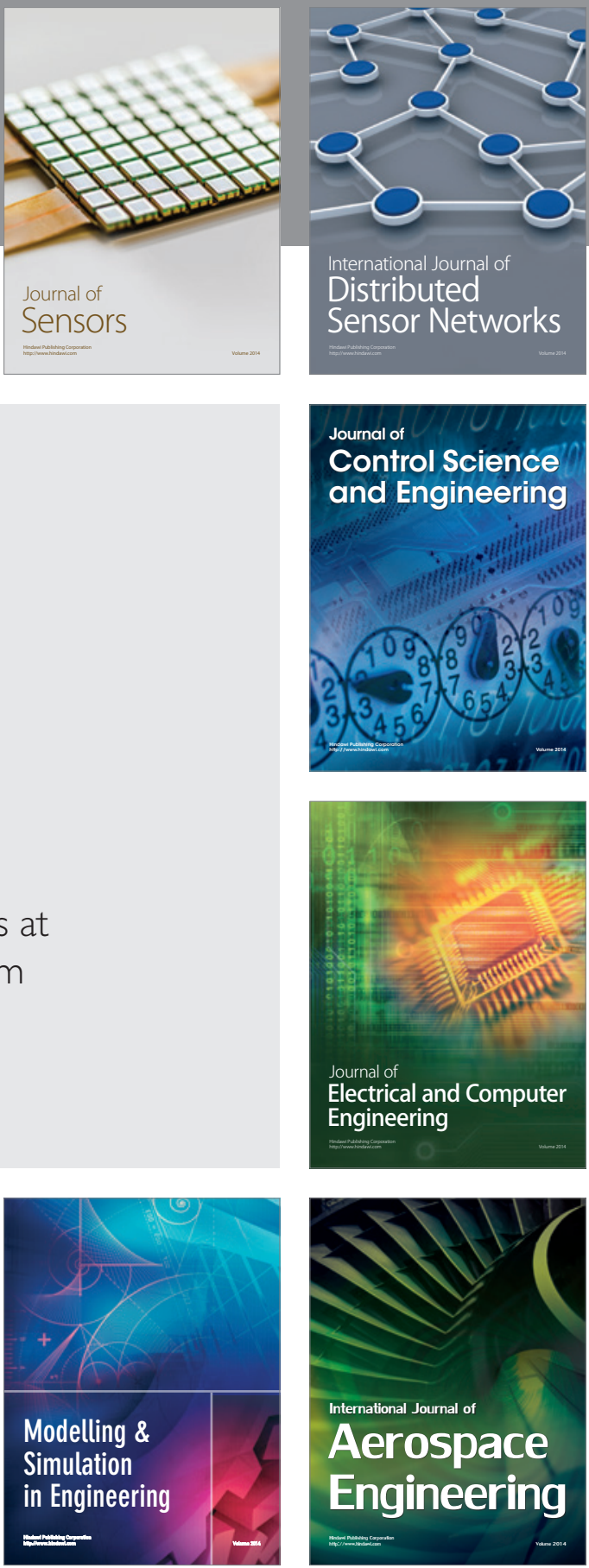

Journal of

Control Science

and Engineering
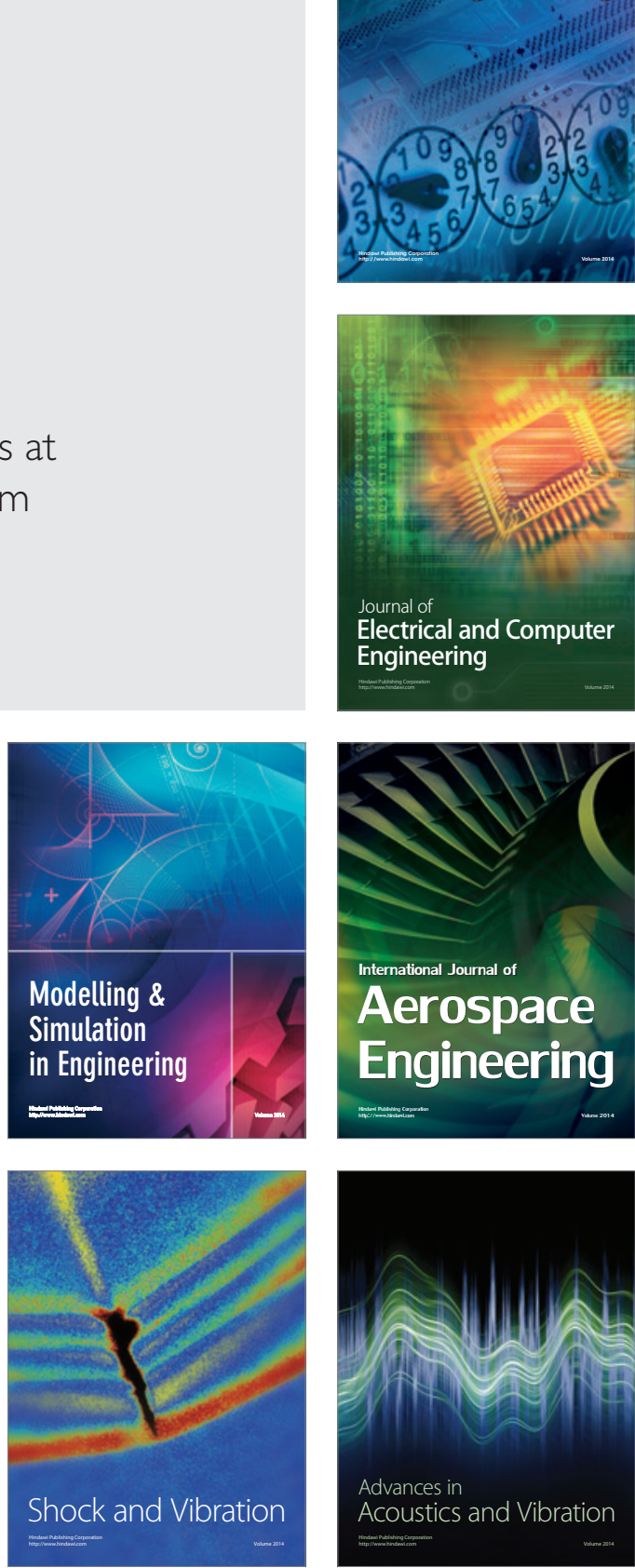